\title{
JULIETTE SIBON
}

\section{Échanges de pratiques et de savoirs entre médecins juifs et chrétiens à Marseille au XIV ${ }^{\mathrm{e}}$ siècle}

L'approche philologique des manuscrits hébraïques produits en Italie et en Provence médiévales, ainsi que l'étude de leurs colophons, révèle combien les juifs de ces deux aires participaient, au bas Moyen Âge, de la sphère culturelle latine occidentale, et combien les liens entre pensées juive et chrétienne étaient étroits ${ }^{1}$. Parmi les intellectuels juifs bien connus en Italie qui participaient de la même dynamique culturelle que les intellectuels chrétiens - car, dans ce domaine, il faut sans doute plutôt évoquer des connivences et des échanges intellectuels que des transferts culturels à proprement parler-, Emmanuel de Rome, adaptateur de l'œuvre de Dante en hébreu, offre un exemple remarquable de savant juif qui cultivait de multiples liens avec la culture chrétienne. Il fréquentait Vérone vers 1328, pôle de contacts entre lettrés juifs et chrétiens, où il échangeait d'ailleurs des poèmes écrits en langue vulgaire avec le poète chrétien italien Bosone da Gubbio ${ }^{2}$. L'Italie attira d'ailleurs des lettrés juifs provençaux familiers avec la culture latine, tel, au XIII ${ }^{\mathrm{e}}$ siècle, Jacob de Lattes, qui comptait parmi ses amis de nombreux médecins chrétiens. Il quitta le sud de la France pour se rendre à Venise, où il traduisit un traité d'astronomie du latin en hébreu³.

Les connivences et les échanges culturels entre lettrés chrétiens et juifs en Méditerranée occidentale sont également perceptibles dans les documents de la pratique, notamment dans les testaments et les inventaires de bibliothèques relevés dans la documentation notariale et judiciaire provençale du $\mathrm{Xv}^{\mathrm{e}}$ siècle, en particulier par Louis Stouff, Noël Coulet et Danièle Iancu${ }^{4}$. Par exemple, la famille juive arlésienne bien connue

1 Ces aspects ont fait l'objet d'un colloque international intitulé »Latin into Hebrew: The Transfer of Philosophical, Scientific and Medical Lore from Christian to Jewish Cultures in Southern Europe (12th-15th Centuries)«, organisé par Resianne Fontaine (université d'Amsterdam) et Gad Freudenthal (Paris, CNRS), et qui s'est tenu les 7, 8 et 9 décembre 2009 à l'université de Paris 7.

2 Emanuele Coccia, Sylvain PIron, Poésie, sciences et politique. Une génération d'intellectuels italiens (1290-1330), dans: Revue de synthèse 129/4 (2008), p. 551-586.

3 Robert CHAZAN, The Letter of R. Jacob ben Elijah to Friar Paul, dans: Jewish History 6 (1992), p. 51-63.

4 Noël Coulet, Un juif d'Aix-en-Provence au début du XV ${ }^{\mathrm{e}}$ siècle. Autour de l'inventaire après décès d'Abram Boniaqui (1424), dans: Danièle IANCU-AGOU (dir.), L'expulsion des juifs de Provence et de l'Europe méditerranéenne: exils et conversions ( $\mathrm{XV}^{\mathrm{e}}-\mathrm{XVI}^{\mathrm{e}}$ siècles), Paris, Louvain 2005, p. 3-12, Louis STOUFF, Isaac Nathan et les siens. Une famille juive d'Arles des XIV et $X^{\mathrm{e}}$ siècles, dans: La famille au Moyen Âge, Provence historique 37/150 (1987), p. 499-512; Danièle IANCU-AgOU, Les œuvres traduites des médecins montpelliérains dans les bibliothèques des juifs du midi de la France au XV siècle, dans: Daniel LE BLÉVEC (dir.), L'uni- 
des Borrian possédait dans sa bibliothèque des commentaires d'Avicenne sur parchemin traduits en latin 5 . On peut encore citer l'exemple de maître Bonet Astruc de Lattes, plus récemment mis au jour par Danièle Iancu, Michele Luzatti et Anna Esposito, qui, dans la dernière décennie du siècle, quitta Aix-en-Provence pour Rome, où il devint médecin et astronome des papes Alexandre VI, Jules II et Léon X. Il fit venir à lui des lettrés chrétiens, et Rabelais lui-même le cita dans son »estude« de l'enfant géant ${ }^{6}$ !

Dans le paysage intellectuel méridional des derniers siècles du Moyen Âge, la communauté juive de Marseille était loin d'être effacée. Dès le début du XII siècle au moins, elle a compté des intellectuels et des personnalités rabbiniques remarqués. Vers 1165 , rappelons-le, Benjamin de Tudèle décrivait le port provençal comme la »ville des géonim [littéralement, des >éminences ‘] et des savants « et admirait son école talmudique $^{7}$. La centralité intellectuelle des juifs de Marseille perdura au-delà du XII ${ }^{\mathrm{e}}$ siècle. $\mathrm{Au} \mathrm{XIII}^{\mathrm{e}}$ siècle naquit le célèbre Moïse ibn Tibbon, traducteur de Maïmonide, et qui vécut à Marseille dans les années 1240-1250. Au XIV siècle, Samuel de Marseille, né à Marseille en 1294 et où il apprit les sciences profanes et la philosophie, traduisit des ouvrages scientifiques arabes en hébreu, dont les commentaires d'Averroès de l'»Éthique« et de la »République«. Notons que l'original du commentaire sur la »République« de Platon ayant été perdu, ce fut à partir de la traduction hébraïque de Samuel que put être élaborée la version latine padouane. Samuel traduisit aussi Euclide, Ptolémée, Alexandre d'Aphrodisie - à partir de sa version arabe, et non grecque -, et d'autres traités d'astronomie8.

Le rayonnement intellectuel de Marseille se pérennisa donc tout au long du $\mathrm{XIV}^{\mathrm{e}}$ siècle. Vers 1320, David Kokhabi, natif d'Étoile, auteur du »Migdal David« et du

versité de médecine de Montpellier et son rayonnement ( $\mathrm{XIII}^{\mathrm{e}}-\mathrm{XV}^{\mathrm{e}}$ siècles), Turnhout 2004, p. $295-305$.

5 Danièle IANCU-AgOU, L'inventaire de la bibliothèque et du mobilier d'un médecin juif d'Aixen-Provence au milieu du XV ${ }^{\mathrm{e}}$ siècle, dans: Revue des études juives 134/1-2 (1975), p. 47-80.

6 RABELAIS, Gargantua, XXI, 110, cité dans: Danièle IANCU-AGOU, Vie privée et réussite sociale dans l'aristocratie juive et néophyte aixoise à la fin du Moyen Âge, dans: Famille et parenté dans la vie religieuse du Midi $\left(\mathrm{XII}^{\mathrm{e}}-\mathrm{XV}^{\mathrm{e}}\right.$ siècles), Toulouse 2008 (Cahiers de Fanjeaux, 43), p. 373-395; EAD. Voix d'exilés et chemins d'errances pour les juifs du Languedoc et de Provence (XIV ${ }^{\mathrm{e}}-\mathrm{XV}^{\mathrm{e}}$ siècles), dans: Jean BALSAMO, Chiara LASTRAIOLI (dir.), Chemins de l'exil, havres de paix. Migrations d'hommes et d'idées au XVI siècle, Paris 2010, p. 13-30. Sur le rayonnement intellectuel de Bonet à Rome, Jean-Claude MARGOLIN, Bonet de Lattes, médecin, astrologue et astronome du pape, dans: Ecumenismo della cultura, vol. 3: L'unanesimo e l'ecumenismo della cultura. Atti del XIV Convegno internazionale del Centro di Studi Umanistici, Florence 1981, p. 107-148.

7 BenJAMin DE TUdĖLE, The Itinerary of Benjamin of Tudela, éd. Marcus Nathan AdLER, Londres 1907, p. 4-5.

8 Adolphe Neubauer, Documents inédits, dans: Revue des études juives 9 (1885), p. 215, Henri GRoss, Gallia judaica. Dictionnaire géographique de la France d'après les sources rabbiniques, Paris 1897, réédition avec suppléments par Simon SchwARZFUCHS, Amsterdam 1969, p. 379, Ernest RENAN, Adolphe NeUBAUER, Les écrivains juifs français au XIV ${ }^{\mathrm{e}}$ siècle, dans: Histoire littéraire de la France 31 (1893), p. 351-830, ici p. 554-567; Lawrence V. BERMAN, Greek into Hebrew: Samuel ben Judah of Marseilles, Fourteenth Century Philosopher and Translator, dans: Alexander AltmanN (dir.), Jewish Medieval and Renaissance Studies, Cambridge 1967, p. 289-350. 
»Kyriat Sefer«, décrivait à son tour la ville comme »un grand centre d'études talmudiques«. En 1408, Isaac ben Sheshet Barfat, connu sous l'acronyme de Ribash, considérait maitre Bonjuson Bondavin comme un grand talmudiste et une sommité rabbinique9. Bonjuson était l'arrière-petit-fils de Bondavin de Draguignan, célèbre prêteur juif marseillais de la première moitié du XIV ${ }^{\mathrm{e}}$ siècle, immortalisé par Joseph Shatzmiller ${ }^{10}$. Médecin et rabbin de Marseille, Bonjuson partit s'implanter en Sardaigne, en 1389, où il exerça la fonction de grand rabbin.

Au-delà des témoignages littéraires, la centralité intellectuelle de Marseille au $\mathrm{XIV}^{\mathrm{e}}$ siècle se dévoile également par le grand nombre de médecins juifs qui apparaissent dans les documents notariaux et judiciaires au sein d'une frange élargie de notables juifs, dans une communauté estimée par Édouard Baratier entre 1000 et 2000 individus, soit environ $10 \%$ de la population totale de la ville. La part des médecins juifs dans la communauté était presque aussi importante que celle des grands prêteurs juifs ( $4 \%$ contre $5 \%$ ). Ces notables, dans l'ensemble, fondaient leur prestige et leur fortune sur le prêt à intérêt, le commerce maritime et terrestre, et l'investissement foncier. L'analyse de leurs activités économiques, et en particulier du circuit des capitaux qu'ils drainaient dans le cadre du prêt à intérêt, dévoile des réseaux d'amitié forte et pérenne de part et d'autres des frontières communautaires, des solidarités entre riches entretenues avec les représentants du patriciat urbain chrétien ${ }^{11}$.

De manière beaucoup plus discrète, un autre réseau de relations entre juifs et chrétiens apparaît dans la documentation latine marseillaise du XIV ${ }^{\mathrm{e}}$ siècle. Ce réseau est en marge, voire déconnecté des réseaux économiques qui viennent d'être évoqués, et est à placer dans le cadre de la pratique médicale et de la circulation des manuscrits. Certes, le fonds marseillais du XIV ${ }^{\mathrm{e}}$ siècle ne recèle pas de beaux inventaires de bibliothèques tels que ceux livrés par les registres aixois et arlésiens du siècle suivant. Il livre toutefois des documents épars, rares et précieux, sur le dialogue savant et la coopération médicale pratique entre les médecins juifs et leurs confrères chrétiens.

\section{LES MÉDECINS JUIFS DANS L'ENVIRONNEMENT CHRÉTIEN PROVENÇAL}

Le recensement des juifs à partir des registres notariés et judiciaires permet d'identifier au moins 20 médecins juifs dans la première moitié du siècle et 38 dans la seconde. Grâce aux chiffres antérieurement établis par Louis Barthélemy au XIX ${ }^{\mathrm{e}}$ siècle et repris par Danielle Jacquart, on connaît donc à Marseille 20 médecins juifs pour 13 chrétiens

9 Isaac Bloch, Bonjusas Bondavin, dans: Revue des études juives 8 (1884), p. 280-283.

10 Joseph Shatzmiller, Shylock revu et corrigé. Les juifs, les chrétiens et le prêt d'argent dans la société médiévale, Paris 2000; Juliette SIBON, Bondavin revisité. Le prêteur juif de Marseille Bondavin de Draguignan (v. 1285-1361), suite et fin, dans: Le Moyen Âge, à paraître.

11 Juliette SIBOn, Les juifs de Marseille au XIV e siècle, Paris 2011. 
entre 1300 et 1348, et 38 médecins juifs pour 35 chrétiens entre 1348 et $1400^{12}$. Notons toutefois que les données relatives aux médecins chrétiens ne sont sans doute pas définitives. Une étude prosopographique des praticiens chrétiens à partir des sources latines marseillaises permettrait assurément une mise à jour. Pour l'heure, on retient la surreprésentation des médecins juifs dans la communauté et leur poids numérique dans la société urbaine marseillaise en général.

Pour la seconde moitié du XIV ${ }^{\mathrm{e}}$ siècle, on peut reconstituer deux dynasties médicales juives sur plusieurs générations, celles des Palerme et des Marvan, avec, pour la première, Mossé de Palerme, mort en 1352, fils de maître Salomon et petit-fils de maitre Samuel, et, pour la seconde, Ferrier Marvan et Marvan Ferrier. Si ces deux familles attestent la transmission verticale du savoir médical de génération en génération, il semble avoir été également possible pour les juifs d'y accéder sans être issu d'une puissante dynastie de praticiens et, qui plus est, sans connaître une carrière plus tard terne ou secondaire. Tels étaient les cas de maître Bonjuson Bondavin (v. 1355-v. 1408), ainsi que de maître Abraham Bondavin d'Avignon (recensé 17 fois dans la documentation latine marseillaise entre 1369 et 1402), issu de la célèbre famille arlésienne des Orgier. La documentation latine ne laisse apparaître dans leur ascendance aucun praticien ayant exercé à Marseille. Tous deux étaient liés par alliance: le père de maître Abraham avait épousé Bella, grand-tante de Bonjuson. Tous deux étaient issus de familles prestigieuses sur le plan économique et se sont retrouvés à la tête de fortunes restées compactes, faute d'héritiers pléthoriques.

Au bas Moyen Âge, l'accès à l'université était théoriquement interdit aux étudiants juifs. Devenus médecins, il leur était alors formellement proscrit de prodiguer leurs soins à des patients chrétiens. Par exemple, la législation de Charles II, en 1306, puis celle du concile d'Avignon de 1337, interdisaient à tout chrétien, quel que fût son statut, de solliciter les services d'un médecin juif, si éminent fût-il. De surcroît, interdiction formelle était faite aux étudiants et aux médecins chrétiens de donner ou de vendre des livres de médecine à un juif, sous peine d'excommunication ${ }^{13}$.

Or la norme discriminante était contournée par les pouvoirs eux-mêmes, dans le cadre de l'expertise médicale notamment. On connaît les exemples manosquins de Shatzmiller ${ }^{14}$. Le fonds marseillais en livre de similaires, telle la »depositio medicorum « faite par le chirurgien juif Mossé Salves le 7 mars 1403, dans le cadre d'une procédure criminelle, qui fait état de sa collaboration avec le barbier chrétien Guillaume Mathole au cours de l'examen du cadavre d'un chrétien qui présentait 14 blessures, tant à la tête que sur le reste du corps ${ }^{15}$. En outre, la municipalité marseillaise employait des méde-

12 Louis BARTHÉLÉMY, Les médecins à Marseille avant et pendant le Moyen Âge. Discours de réception à l'académie de Marseille, Marseille 1883; Danielle JACQUART, Le milieu médical en France du XII ${ }^{\mathrm{e}}$ au XV ${ }^{\mathrm{e}}$ siècle, Genève 1981; SIBON, Les juifs de Marseille (voir n. 11).

13 Joseph Shatzmiller, Jews, medicine and Medieval Society, Berkeley, Los Angeles 1994, p. 91-92.

14 ID., Médecine et justice en Provence médiévale. Documents de Manosque, 1262-1348, Aix-enProvence 1989.

15 Archives départementales des Bouches-du-Rhône (désormais AD13), 3B, 140. 
cins juifs dans les hôpitaux. Par exemple, maître Ferrier Marvan exerça au service des malades des hôpitaux de l'Annonciade en 1389 et du Saint-Esprit en 139716.

En outre, Shatzmiller l'a souligné, la formation des médecins juifs n'était ni incomplète ni plus médiocre que celle de leurs confrères chrétiens ${ }^{17}$. Depuis juillet 1272 , la licencia practicandi était un diplôme reconnu en Provence, qui autorisait les juifs à exercer la médecine dans les limites du comté. L'exemple provençal le plus célèbre reste celui de Salomonet Avigdor, à qui la licence fut accordée à Arles le 15 mai 1402 par le représentant du viguier royal, après que Salomon eut soutenu publiquement une thèse en présence de quatre médecins, dont un chrétien et trois juifs ${ }^{18}$. En l'absence d'un corps enseignant spécialisé et institutionnalisé, la transmission du savoir était prise en charge par une personne qualifiée, qui rassemblait autour d'elle un groupe d'élèves ${ }^{19}$. Un seul exemple connu subsiste pour le $\mathrm{XIV}^{\mathrm{e}}$ siècle marseillais, révélé par un contrat d'apprentissage, daté du 28 août 1326, dans lequel le juif de Salon-deProvence Salvet de Bourgneuf, fils de feu Davin de Bourgneuf, se déclarait élève de Sarah de Saint-Gilles, épouse d'Abraham de Saint-Gilles, et s'engageait à rester à son service afin d'apprendre à ses côtés »l'art de la médecine«, tandis que Sarah promettait de bien le former ${ }^{20}$. L'exemple atteste la renommée de Sarah, et peut-être des médecins juifs marseillais en général, bien au-delà des murs de la ville, au moins jusqu'à Salonde-Provence.

La diffusion du savoir médical était d'ailleurs étroitement contrôlée au sein des élites intellectuelles juives. À Avignon, au XIV ${ }^{\mathrm{e}}$ siècle, les chirurgiens juifs enseignaient leur art à condition que leurs élèves aient accepté de ne pas exercer pendant une période de deux à huit ans. Il s'agissait, pour ces maîtres, de se protéger de la concurrence future de leurs émules ${ }^{21}$. Un contrat marseillais daté de 1316, analysé par Shatzmiller, confirme que le savoir médical n'était pas généreusement partagé. Il s'agit de la location pour un an du »Sefer ha-Zahrawi«, ouvrage d'Abū 'l-Qāsim Halaf az-Zahrāwī (XII ${ }^{\mathrm{e}}$ siècle) traduit en hébreu à Marseille au milieu du XIII ${ }^{\mathrm{e}}$ siècle par Shem Tob ben Isaac de Tortose, et qui était alors considéré comme le livre de base de l'étude de la médecine ${ }^{22}$. Le manuscrit appartenait à Mosson, fils de maître Abraham, qui exigeait la

16 Augustin FABRE, Histoire des hôpitaux et des institutions de bienfaisance de Marseille, Marseille 1854 , vol. 1, p. 192-193.

17 ShatzMiller, Jews, Medecine and Medieval Society (voir n. 13).

18 Voir notamment Paul HiLDENFINGER, Documents relatifs aux juifs d'Arles, dans: Revue des études juives 41 (1900), p. 67.

19 Simon SchWARZFuchS, Études sur l'origine et le développement du rabbinat au Moyen Âge, Paris 1957.

20 AD13 381 E 32, fol. 122, cité dans: BARTHÉLÉMY, Les médecins à Marseille (voir n. 12), p. 25.

${ }^{21}$ Paul PANSIER, Les médecins juifs à Avignon auX XIII ${ }^{\mathrm{e}}, \mathrm{XIV}^{\mathrm{e}}$ et $\mathrm{XV}^{\mathrm{e}}$ siècles, dans: Janus. Archives internationales pour l'histoire de la médecine et pour la géographie médicale (1910), p. 426427, 440-442.

22 Joseph SHATZMiLleR, Livres médicaux et éducation médicale: à propos d'un contrat de Marseille en 1316, dans: Mediaeval Studies 42 (1980), p. 463-470. 
somme un peu excessive, au regard des prix du marché, de 20 florins ${ }^{23}$. Cette précaution témoigne du contrôle de la diffusion des ouvrages, et donc de l'activité médicale, à laquelle aspiraient les médecins juifs, par l'usage de procédés élitistes, dans le souci de tempérer la concurrence. En effet, Mosson fit des réserves pour limiter la propagation de son texte. L'emprunteur du manuscrit, Bonfils, accepta de le copier uniquement pour lui-même. Il ne fut autorisé à en faire qu'une seule copie, qui ne devait être ni reproduite ni prêtée. La durée de la location fut fixée à un an et pas un jour de plus. Bonfils ne fut autorisé à montrer sa copie qu'à quelques personnes, dont son propre fils, mais pas avant que ce dernier eut atteint l'âge de quinze ans. Enfin, dans le cas où Bonfils mourrait sans héritier, la copie devrait être renvoyée à Marseille et confiée à Mosson. Bonfils accepta toutes ces clauses.

À partir du XIII ${ }^{\mathrm{e}}$ siècle, les juifs non arabophones de Proventsa - transcription du terme hébraïque correspondant au terme latin »Provincia« et qui désigne le Midi de la France - eurent à leur disposition un corpus d'écrits scientifiques en hébreu, composé de Galien, d'Avicenne, de Razès, ainsi que d'Aristote, via Averroès notamment, grâce à l'œuvre des célèbres dynasties de traducteurs languedociens, dont les Tibbonides au premier chef ${ }^{24}$. En outre, il ne faut pas négliger la production des savants juifs euxmêmes, celle de Maïmonide (1138-1204) par exemple, et, avant lui, celle d'Isaac Israeli ( $\mathrm{IX}^{\mathrm{e}}-\mathrm{X}^{\mathrm{e}}$ siècles), dont l'œuvre était au programme de la licence de médecine à Montpellier en 130925 .

D'ailleurs, la faculté de médecine de Montpellier ouvrait ses portes aux médecins juifs provençaux, d'une façon ou d'une autre ${ }^{26}$. Jacob ben Makhir, médecin, astronome et traducteur issu de l'illustre famille des Tibbonides, qui vécut dans la seconde moitié $\mathrm{du} \mathrm{XIII}^{\mathrm{e}}$ siècle, était particulièrement fier des rapports de cordialité et de collaboration qui existaient entre les savants juifs et leurs homologues chrétiens. Lui-même entretenait des relations personnelles avec Bernard de Gordon et Arnaud de Villeneuve. Ce dernier, natif de Catalogne, menait des affaires à Montpellier comme à Marseille27. Il existait un axe entre les deux villes pour les savants juifs et leurs contemporains chré-

23 Shatzmiller a insisté sur la dimension lucrative des précautions prises par le propriétaire du manuscrit, en émettant l'hypothèse que Mosson était un descendant de Shem Tob et s'assurait ainsi des >droits d'auteur< sur le manuscrit dont il aurait hérité.

24 Gad Freudenthal, Les sciences dans les communautés juives médiévales de Provence: leur appropriation, leur rôle, dans: Revue des études juives 152 (1993), p. 29-136; EAD. Transfert culturel à Lunel au milieu du XII ${ }^{\mathrm{e}}$ siècle: Qu'est-ce qui a motivé les premières traductions provençales de l'arabe en hébreu?, dans: Danièle IANCU-AgOU, Élie Nicolas (dir.), Des Tibonnides à Maïmonide. Rayonnement des juifs andalous en pays d'Oc médiéval, Paris 2009, p. $95-108$.

25 En janvier 1181, Guilhem VIII avait déclaré la liberté d'enseignement de la médecine, faisant de Montpellier le siège d'un savoir médical véhiculé par des maîtres de tous les horizons. Voir Danièle IANCU, Carol IANCU, Les juifs du Midi. Une histoire millénaire, Avignon 1995, p. 57.

26 Joseph Shatzmiller, La faculté de médecine de Montpellier et son influence en Provence: témoignages en hébreu, en latin et en langue vulgaire, dans: LE BLÉVEC (dir.), L'université de médecine de Montpellier (voir n. 4), p. 291-294.

27 Donatella NeBbiai DE LA GuARdia, Un intellectuel catalan à Marseille: Arnaud de Villeneuve, dans: Thierry PÉCOUT (dir.), Marseille au Moyen Âge, entre Provence et Méditerranée. Les horizons d'une ville portuaire, Paris 2009, p. 340-343. 
tiens. Ainsi, à la fin du XIV siècle, le médecin juif provençal Léon Joseph de Carcassonne, qui traduisit des traités de médecine du latin en hébreu, se vit accorder l'accès à l'université de Montpellier par le chancelier Tournemire28.

Dans ce contexte, nul doute que les manuscrits circulaient entre juifs et chrétiens. Bien qu'on ne connaisse pas les détails de l'affaire, le procès en date du 21 avril 1374, qui opposa Bonet, habitant juif d'Avignon, à Vidonet Bonisac, juif de Marseille, confirme que la détention d'ouvrages médicaux pouvait donner lieu à d'âpres disputes ${ }^{29}$. Le premier réclamait au second 21 volumes hébraïques que lui avait commandés maître Jean Algen, médecin chrétien d'Avignon. La peine fixée par le tribunal atteignait alors la somme élevée de 200 livres. On ignore tout des volumes en question: étaient-ils des ouvrages de médecine que Algen comptait utiliser personnellement? L'hypothèse interpelle dans la mesure où, en théorie, les médecins chrétiens n'avaient rien à envier à la connaissance médicale des médecins juifs. La deuxième question qui se pose au sujet de ces ouvrages est celle de leur langue. Si l'hébreu était la langue de l'apprentissage médical pour les médecins juifs, les médecins chrétiens puisaient leur savoir dans des manuscrits latins. Ces questions restent sans réponses. L'affaire démontre néanmoins que des transferts existaient entre le milieu médical juif et le milieu médical chrétien, et que les manuscrits circulaient d'un groupe à l'autre. Il se pourrait même que Jean ait travaillé en étroite association avec un confrère juif, seul susceptible de lui révéler le contenu des ouvrages hébraïques. À moins - et c'est la dernière hypothèse - que Jean fût néophyte.

\section{ÉCHANGES THÉORIQUES ET PRATIQUES ENTRE MÉDECINS JUIFS ET CHRÉTIENS}

La preuve la plus tangible et la plus riche d'échanges de savoir et de pratiques entre médecins juifs et chrétiens à Marseille au XIV ${ }^{\mathrm{e}}$ siècle est livrée par un procès exhumé pour la première fois par Félix Portal en 1902, qu'il convient de reprendre et d'analyser plus avant ${ }^{30}$. Le procès implique maître Abraham Bondavin d'Avignon, déjà évoqué plus haut. Citoyen juif de Marseille, il épousa la fille de l'un des grands hommes d'affaires juifs marseillais du XIV siècle, dénommé Anthol Samson, et il occupa des responsabilités à la tête de la communauté, en tant que syndic notamment, en 1388-1389 puis en 1399-1400.

Le 15 novembre 1389, il comparaissait devant la Cour du palais - tribunal angevin sis à Marseille - pour répondre d'une accusation, sinon de charlatanisme, du moins d'erreur médicale ${ }^{31}$. Il fut condamné en première instance à une amende de 25 livres de

28 Salomon KAHN, Les écoles juives et la faculté de médecine de Montpellier, Montpellier 1890.

29 AD13 3B 86, fol. 33.

30 Félix PORTAL, Un procès en responsabilité médicale, Marseille 1902, cité par: Adolphe CRÉMIEUX, Les juifs de Marseille au Moyen Âge, dans: Revue des études juives 46 (1903), p. 1-47, ici p. 42.

31 AD13 3B 844, fol. 47v-95v. 
royaux d'une part, pour avoir prescrit un mauvais traitement, et de 10 livres de royaux d'autre part, pour avoir exercé illégalement la chirurgie. Au moment du procès, Abraham était, en effet, qualifié en médecine et non en chirurgie. Il avait prescrit à l'un de ses patients chrétiens, le pêcheur Aycardet André que l'on croyait, dans un premier temps, atteint de la lèpre, une ordonnance qui aurait eu pour conséquence de rendre le malade impotent pour le reste de ses jours. Bien que les évêques se soient vus confier très tôt la tâche d'assister les lépreux ${ }^{32}$, et que les léproseries se soient multipliées au cours des $\mathrm{XII}^{\mathrm{e}}$ et XIII ${ }^{\mathrm{e}}$ siècles, les médecins juifs étaient sollicités à Marseille au XIV ${ }^{\mathrm{e}}$ siècle pour tenter de trouver des solutions au mal réputé contagieux et incurable ${ }^{33}$.

Pourquoi a-t-on douté des compétences d'Abraham Bondavin d'Avignon en 13891390? Le choix des experts sollicités par la Cour pour juger de sa pratique écarte d'emblée toute idée de discrimination à l'encontre des juifs. Il s'agit du médecin chrétien maître Lambertin Ramaudon et des deux médecins juifs maître Dieulocrescas Jossé Roget, qui est dit »medicus physicus «, et maître Ferrier Marvan, chirurgien issu d'une longue lignée de médecins, on l'a vu. Tous trois s'appuient sur »leur science et leur pratique« qui les conduisent à des observations identiques, en dépit de leur différence de spécialité. Le suc d'ail et le suc d'ortie dite >barbue pour fabriquer l'onguent appliqué sur la peau d'Aycardet ne sont pas préconisés dans le traitement de la lèpre. Tous deux sont "chauds et corrosifs« et donc très nocifs. Dieulocrescas et Ferrier connaissaient la théorie des corps, des plantes et des simples. Leur lexique révèle le cadre doctrinal auquel ils se référaient les uns comme les autres, à savoir celui de l'humorisme hippocratique, qui consistait à rétablir l'équilibre entre les quatre humeurs ou qualités élémentaires du corps humain (le chaud, le froid, le sec et l'humide). En vertu de cette doctrine, les causes attribuées aux troubles cutanés, dont la lèpre, étaient celles de l'excès de certaines humeurs. En conséquence, la lèpre était souvent traitée par l'emploi du sirop de fumeterre aux propriétés apéritives, c'està-dire qui dilataient les vaisseaux afin de faciliter la circulation des humeurs ${ }^{34}$.

Pourtant, la première thérapie prescrite à Aycardet fut chirurgicale. En effet, Abraham ne fut appelé que dans un second temps auprès d'Aycardet. Ce fut le chirurgien juif Vital de Lunel qui prodigua les premiers soins. Il apposa des fers chauds sur les plaies du patient et lui amputa des os des mains et des pieds. Il jugea que la cause principale des plaies et des souffrances du patient était le froid. Par conséquent, il voulut sans doute rétablir l'équilibre des humeurs par la chaleur.

Puis maitre Abraham intervint. Le procès révèle qu'il collaborait avec un médecin chrétien, maître Raymond Pibarot ${ }^{35}$, décédé au moment du procès, et avec un apothi-

32 Dès le concile d'Orléans de 549.

33 Mirko D. GRMEK, Histoire de la pensée médicale en Occident, vol. 1: Antiquité et Moyen Âge, Paris 1993, p. 302, 307.

34 Jean-Pierre BÉNÉZET, Pharmacie et médicament en Méditerranée occidentale (XIII ${ }^{\mathrm{e}}-$ $\mathrm{XVI}^{\mathrm{e}}$ siècles), Paris 1999, p. 579.

35 Originaire du diocèse de Nîmes, il obtint son diplôme de médecin à Montpellier en 1378 et est connu comme praticien à Marseille en 1382. Voir Ernest WICKERSHEIMER, Dictionnaire biographique des médecins en France au Moyen Âge, Genève 1979, p. 678. 
caire chrétien, Jacques Ferrand, alias Galhart ${ }^{36}$. Or, les détails du procès révèlent plus que des transferts de savoirs et de pratiques entre les deux confrères, juif et chrétien. Leur coopération s'étend à la promotion et à la mise en pratique d'une thérapie innovante, dont l'audace est sans doute à l'origine du procès. Cette thérapie s'émancipe, sinon de la théorie hippocratique, du moins de la façon dont les trois experts médicaux la concevaient et l'appliquaient.

La thérapie mise en œuvre par Abraham et Raymond fut contestée, y compris par son patient lui-même, dont les paroles, rapportées par ses proches et proférées dans d'affreuses douleurs, n'expriment cependant aucune discrimination. Après avoir hurlé »Halas! Jeu crevi tot! Jeu crevi tot! «, Aycardet ajoute en substance: »Je meurs et tu es le faux médecin (falsus medicus) qui me tue! « Le terme »falsus« est riche de nuances. Il qualifie le menteur et l'hypocrite, mais aussi le pervers et le traitre! Abraham avait commandé à l'apothicaire Jacques Ferrand la fabrication d'un onguent - sans doute dans le but de désinfecter les plaies d'Aycardet - composé d'une grande quantité de sucs d'ail et d'ortie (»sucus alhiis et sucus urticarum barbanarum«), qu'il avait ensuite appliqué sur tout le corps du patient, des pieds à la tête. On peut admettre que l'acidité du baume ait provoqué des sensations de brûlures chez le patient mais, si »corrosif et chaud « fût-il, aux dires des experts médicaux entendus, on doute qu'il ait pu contribuer à rendre Aycardet impotent pour le restant de ses jours! D'ailleurs, face aux cris de douleur du malade, Abraham eut le réflexe immédiat de proposer qu'on lui prépare un bain pour enlever la pommade et le soulager.

En conséquence, comment interpréter l'hostilité de Ferrier Marvan, de Dieulocrescas Jossé Roget et de Lambertin Ramaudon? S'agit-il d'un règlement de comptes entre praticiens concurrents? Bien que Ferrier Marvan ait émis un jugement catégorique sur la prescription d'Abraham en première instance, il n'hésita pas à témoigner une seconde fois en appel afin de le disculper de l'accusation d'avoir illégalement exercé la chirurgie. Il attesta que les amputations avaient été réalisées, non par Abraham, mais par le chirurgien Vital de Lunel. Si l'expertise des trois confrères d'Abraham conclut à l'erreur médicale, ce ne fut donc pas à la suite de manipulations malhonnêtes et calomnieuses. De bonne foi, ils estimèrent qu'Abraham s'était écarté de la doctrine humoriste ancestrale. Or, pour sa défense, Abraham ne nia pas la composition de l'onguent qu'il avait commandé au pharmacien chrétien. Au contraire, il s'attacha à prouver le bien-fondé scientifique de sa pratique. Il rappela tout d'abord qu'il était un homme honnête (»homo bone fame et honeste conversationis«) qui s'était toujours abstenu de commettre des actes illicites, ce qui était de notoriété publique dans son voisinage. De surcroît, il était devenu médecin à Marseille sept ou huit ans auparavant, après qu'il eût prêté le serment d'exercer la médecine »bene et fideliter«. En effet, il avait obtenu la licencia practicandi valable dans tout le comté de Provence, et était donc un savant reconnu (»approbatus scientificus in arte medicine«). Enfin, il précisa que l'onguent avait été conçu en accord avec son confrère chrétien maître Raymond Pibarot, qui avait aussi la réputation d'être un savant honnête et un expert en médecine (»bonus homo legalis scientificus et expertus in arte medicine«).

36 On ignore tout sur ce dernier. 
La bonne réputation des deux médecins semble, en effet, bien établie dans la cité. Pour preuve, Abraham convoqua à la barre des témoins des représentants du patriciat urbain chrétien marseillais. Ce dernier était composé de nobles non fieffés, enrichis par le commerce dès les $\mathrm{XII}^{\mathrm{e}}$ et $\mathrm{XIII}^{\mathrm{e}}$ siècles, qui arboraient des titres de noblesse - ceux de nobiles ou de miles -, et qui étaient de gros propriétaires fonciers, au faîte de l'administration communale. Il incluait aussi des marchands de notoriété plus récente, également membres du conseil municipal. Les premiers furent ici représentés par le noble Pierre de Servières, qui occupait la fonction de recteur de l'hôpital des pauvres du Christ et du Saint-Esprit, où - on l'apprend dans le procès - maître Abraham prodiguait également ses soins; les seconds par le marchand Pierre Daumas.

Le rôle de maitre Abraham en tant qu'acteur de transferts culturels en direction de la majorité chrétienne se fondait donc sur la bona fide dont le créditait la société urbaine marseillaise, et les élites marseillaises en particulier. Abraham était reconnu pour les compétences médicales dont il avait largement fait les preuves, ainsi que pour le respect de la légalité dont il pouvait s'honorer: il n'avait pas exercé la chirurgie illégalement; il avait prodigué des soins en vertu du diplôme reconnu par le sénéchal de Provence, représentant du comte; il avait fait préparer l'onguent chez un apothicaire, dans le respect le plus strict des statuts de la ville de Marseille.

\section{CONCLUSION}

On peut donc conclure à une forme de créativité médicale dans la pratique d'Abraham, fruit de sa collaboration avec un confrère chrétien. Le procès de maitre Abraham Bondavin d'Avignon, enrichi par les données prosopographiques recensées dans le reste de la documentation notariale marseillaise du XIV siècle, ne dévoile pas des transferts inégaux de médecins chrétiens à médecins juifs, à savoir d'un pôle documenté vers un pôle moins documenté. La collaboration entre maître Abraham et maître Raymond Pibarot relève du partage d'intérêts scientifiques communs, mis en pratique au quotidien.

De surcroît, le clivage qui apparait entre Abraham et Raymond d'une part, et Ferrier, Dieulocrescas et Lambertin d'autre part, est bel et bien scientifique. Face à des expérimentations innovantes, se dressent les tenants d'une pratique de la médecine plus conservatrice, qui considèrent toute entorse à leur conception de la théorie des simples comme une erreur médicale.

Pour autant, le désaccord entre maître Abraham et les experts médicaux ne semble pas s'être étendu au-delà du terrain scientifique. Le 2 septembre 1397, en effet, Abraham épousait en secondes noces Durante, la fille de maitre Ferrier Marvan, dont la dot s'élevait à 376 florins. En outre, les ennuis de maître Abraham semblent s'être vite résolus. L'issue de l'appel manque, mais la documentation postérieure montre qu'il obtint finalement gain de cause. En dépit des graves accusations dont il avait fait l'objet en 1389, il fut à nouveau sollicité quelques années plus tard, en avril 1402, avec deux confrères chrétiens, maître Jean de Neufbourg, licencié en médecine, et maître 
Pierre Gaudin, bachelier en médecine, pour examiner Alissona, femme de Jean Davin, et leur fille Dulciette, que l'on craignait, à tort, infectées par la lèpre, et qui étaient alors retenues en quarantaine chez elles, sur ordre du viguier royal, Guillaume Pierre Lascar $^{37}$.

Les sources sont certes rares et dispersées, mais elles dévoilent une dynamique intellectuelle et scientifique qui transcende les frontières communautaires entre juifs et chrétiens au sein du milieu médical provençal du XIV siècle, révélée par la circulation des manuscrits et la coopération pratique, dans le cadre de l'expertise médicale, au sein des hôpitaux ou auprès de patients communs. C'est d'ailleurs au sein de ce milieu médical provençal juif que Danièle Iancu repéra des conversions au christianisme précoces et sincères, dès les années 1460 , dans un contexte tranquille, tandis qu'aucune ombre ne venait encore ternir le destin provençal des juifs médiévaux ${ }^{38}$.

38 Danièle IANCU-AgOU, Juifs et néophytes en Provence. L'exemple d'Aix à travers le destin de Régine Abram de Draguignan (1469-1525), 2 vol., Paris, Louvain 1995. 